\title{
Thermal Modulation Transfer Function (TMTF)
}

\author{
by J.-C. Krapez
}

ONERA, DMSE, BP 72, F-92322 CHÂTILLON-Cedex, France.E-mail: krapez@onera.fr

\begin{abstract}
:
The TMTF is a tool for thermal NDE (pulsed thermography). It gives a synthetic view of the thermal contrast degradation that is inherent with a reduction of the defect size. It was built, like the MTF in visual or IR imaging, by using a target with a periodic pattern. The TMTF was numerically simulated with a model having a cluster of parallel slots on the rear side. The class of slots having a low relative depth is aimed to simulate corrosion at its onset. For this class, the TMTF is expressed as a unique curve that moves with the time of observation. An inversion method based on the TMTF was proposed: it clears, to some extent, the observed temperature field from the detrimental effect of lateral diffusion.
\end{abstract}

\section{Introduction}

A common practice for evaluating the performance of a given lens or optic system, is to measure the image contrast when viewing a group of periodic patterns with different spatial frequencies. As frequency increases, the contrast is reduced due to a variety of factors like diffraction, the limited size of the sensing elements, the limited bandwidth of the electronic system in the case of a scanning instrument... A typical figure of merit of the optic system is the Modulation Transfer Function (MTF), a curve that illustrates the evolution of the contrast vs. spatial frequency. Frequency is given in $\mathrm{mrad}^{-1}$ or sometimes in pixel ${ }^{-1}$.

Our aim is to apply the concept of MTF used in optics to the signal obtained with pulsed stimulated thermography in the field of NDE. The purpose is to derive some general trends regarding the expected thermal contrast when controlling a corroded part. There are many studies, both numerical and experimental, on the temperature evolution in a flawed material, in particular in materials containing holes and cracks. Here we wanted to express thermal diffusion results in a way that is common to the optic system testing area. Some papers tackling the experimental aspect of this problem were recently published [1, 2].

\section{Description of the model}

The numerical model used for this study is a plate with many parallel slots on the rear side (see fig. 1). The bottom of these slots is at a distance $l_{1}$ from the front face (the one receiving the pulse and the one whose temperature field is monitored), their depth is $l_{2}$ (as measured from the rear face), and their width is $d$. Like with the targets that are commonly used in optical resolution measurements, we considered that the duty factor is here 0.5 (the width of the slots is equal to the spacing between them). When compared to optical targets, the "thermal target" has a third dimension: the depth of the periodic structure has to be taken into account. Indeed a series of slots with the same spatial frequency is expected to yield a lower thermal contrast as the distance to the front surface $l_{1}$ increases. Furthermore, the thermal print of the slots on the front surface temperature field is a time evolving function. These considerations lead to the introduction of the following non-dimensional parameters: the relative depth $l_{2} / l_{t}$, the Fourier number $F o_{t}$, and the normalised frequency $f_{t}$ :

$$
\begin{aligned}
& F o_{t}=a_{z} t / l_{t}^{2} \\
& f_{t}=\frac{l_{t}}{2 d} \sqrt{\frac{a_{x}}{a_{z}}}
\end{aligned}
$$


where $l_{t}$ is the total thickness, $a_{x}$ and $a_{z}$ are the in-plane and normal diffusivities (the plate may be anisotropic).

We used a finite difference method, based on the control volume approach and on the alternate direction implicit (ADI) technique [3]. Automatic grid generation with non-uniform node density is generated upon specification of the geometric and thermal parameters of the model: $l_{2} / l_{t}$ and $f_{t}$. The 2D grid contains typically 3600 elements. The computation takes a couple of seconds on a PC $450 \mathrm{MHz}$.

\section{Illustration of typical thermograms and contrast profiles}

We reported in fig. 2 a series of thermograms for a particular configuration of the slot cluster. Apart from the reference thermogram, i.e. the one corresponding to a plate without slots, we plotted the thermograms recorded just above a slot centre, just between two slots, and just above a slot edge. All these three thermograms converge to a common value corresponding to the adiabatic level defined by the material volume (we assumed that there are no heat losses). The temperature above a slot is, of course, the maximum temperature; it will be called $T_{\max }$. Respectively, the temperature between two slots will be written $T_{\min }$. Interestingly, on the surface in the region between two slots, the temperature first drops below the adiabatic level and then increases back to this value. It is the result of a trade-off between heat flow in the $z$-direction towards the rear face, and heat flow in the $x$-direction from the region above the slots to the regions nearby.

The temperature profile, more precisely the contrast $T(x)-T_{\min }$, is plotted in fig. 3 for different values of the Fourier number. The profiles increase in height with the Fourier number and then decrease after a time that depends on the relative depth of the slots. For the considered configurations, the profiles are always very smooth, and there is no indication on the slot edge position (no slope discontinuity at $x / d=0.5$ ).

\section{Modulation Transfer Function}

We defined, like in optics, the transfer modulation function according to:

$$
M=\frac{T_{\max }-T_{\min }}{T_{\max }+T_{\min }}
$$

Naturally, this function evolves with time. It first increases (diffusion in the z-direction and the heat stopping induced by the slot yield a contrast on the front surface), and then decreases (diffusion in the $\mathrm{x}$-direction makes the contrast vanish). The geometric factors: $l_{2} / l_{t}, d / l_{1}$, and eventually the anisotropy factor $a_{x} / a_{z}$, are the parameters that make the Thermal Modulation Transfer Function (TMTF) reach higher or lower values, at earlier or later times.

In fig. 4 we can see the time evolution of the TMTF for two particular values of the relative depth: $l_{2} / l_{t}=0.5$ (50\% material loss, for simulated corrosion) and $l_{2} / l_{t}=0.1$ ( $10 \%$ material loss). A low normalised frequency (wide slots) induces a higher modulation. Simultaneously, the maximum of the function $M\left(F o_{t}\right)$ occurs at larger values of the Fourier number. The onset depends primarily on the relative depth $l_{2} / l_{t}$, not on the normalised frequency $f_{t}$. The plots in fig. 4 highlight again the advantage of using early thermographic data for defect mapping [4]. Indeed, to get information on detailed features, i.e. those with high spatial frequencies, one has to capture images as soon as diffusion reaches the considered objects. Of course, by waiting longer, one would get higher modulation values for those features that contain low frequencies, but this will be at the expense of loosing all information on the smallest details. 
In fig. 5 we plotted the TMTF in another way: the two plots show modulation values as developed at two particular Fourier numbers. We chose a relatively short time: $F o_{t}=0.3$, and a larger one: $F o_{t}=0.5$. All curves indicate how fast the modulation drops when the spatial frequency increases. Furthermore, as mentioned before, by waiting from 0.3 to 0.5 , one gets an improvement for the lowest values of $f_{t}$. However, the modulation is simultaneously lowered for frequencies higher than about 0.5 .

One way to figure how dramatic the lateral diffusion effect is on the defect appearance, is to divide the TMTF by the value pertaining to infinitely low frequency (1D diffusion model). The so-called normalised modulation is plotted in fig. 6, again for two values of the Fourier number: 0.3 and 0.5 . First interesting result is that, in each bundle, the curves nearly overlap. There is thus a kind of general trend for the TMTF of the shallowest slots $\left(l_{2} / l_{t} \leq 0.5\right.$, i.e. those simulating the corrosion at its earliest stage). The normalised TMTF drops from 1 to virtually 0 for a frequency $f_{t}$ between, roughly 0.1 and 1 . At $F o_{t}=0.5$, the normalised TMTF starts to drop for even lower values of $f_{t}$.

Fig. 6 is thus a synthetic figure of the resolution loss induced by lateral diffusion in thermal NDT. Thermal diffusion clearly acts as a low-pass filter. This figure shows in a particular way the well-known characteristic that defects of small extension are difficult to detect by thermal methods.

\section{Inversion}

Once the MTF is obtained, one can try to use it for an inversion purpose, like in visual or IR imaging. Of course, it is here a crude transposition of a procedure that is only valid for a linear process. Here, the "degradation" function is the one that links, as the input, the temperature field on the front surface one would get if lateral diffusion were absent, and as the output, the real, "smooth" temperature field. One can consider the normalised TMTF plotted in fig. 6 as the Fourier transform of this "degradation" function. From that point, one can implement an inversion procedure, by using for example Wiener inverse filtering. Starting from a real temperature field, i.e. a field degraded by lateral diffusion, the inverse filtering is expected to provide temperature distribution deprived from lateral diffusion. Such a virtual field will thus yield a more acute representation of the defect extension. This procedure was applied on the temperature field obtained with the model used in the first part, i.e. a periodic cluster of slots (fig. 7). This "degraded" field is nearly a sinusoidal function at $F o_{t}=0.3$ (high frequencies are highly damped as expected from fig. 6). Without lateral diffusion, one should get a two-level function (a high level above the slots, and a low level in-between). Despite the high damping, the proposed inversion procedure gives interesting results: the slot edges are more clearly revealed (the slope is higher), and the two nominal levels are nearly reached. Some spurious oscillations however appear at higher spatial frequency, but this is inherent to Wiener inverse filtering.

\section{Conclusion}

With a finite difference model, we calculated the temperature field in a plate containing a periodic cluster of slots on the rear side. The plate was assumed to be pulse heated on the front surface. The purpose was to calculate the spatial modulation of the temperature on the front surface. A large series of geometric configurations was considered in order to build the so-called Thermal MTF (TMTF). As compared to the classic optical MTF, specific parameters here appear: a normalised spatial frequency that takes into account a possible thermal anisotropy, the relative depth of the slots, a Fourier number that expresses the time dependence. The TMTF synthetically describes the low pass filter effect that stems from lateral diffusion. We finally proposed an approximate inversion method based on the TMTF. The purpose is to transform the "degraded" surface temperature field into the field one would 
get if lateral diffusion were absent. It was shown on a first example that this virtual field gives a sharper image of the true defect shape.

\section{Acknowledgement}

The author gratefully acknowledges fruitful discussions with S.M. Shepard, Thermal Wave Imaging, Inc, on what we finally called the Thermal Modulation Transfer Function.

\section{References}

[1] SHEPARD, S.M., AHMED, T., and CHAUDHURY, B.C., "Resolution criteria for subsurface features in active thermography", Review of Progress in QNDE, vol. 18, eds. D.O. Thomson and D.E. Chimenti (Plenum, N.-Y., 1988), p 605-610

[2] SHEPARD, S.M., and AHMED, T., "Characterization of active thermographic system performance", SPIE conf., Thermosense XXI, Orlando (FI.), April 1999, vol. 3700, p 388-392

[3] KRAPEZ, J.-C, MALDAGUE, X., and CIELO, P., "Thermographic nondestructive evaluation: Data inversion procedures. Part II: 2-D analysis and experimental results", Res. Nondestr. Eval., vol. 3, 1991, p 101-124

[4] KRAPEZ, J.-C. and BALAGEAS, D., "Early detection of thermal contrast in pulsed stimulated infrared thermography", Quantitative Infrared Thermography (QIRT 94), Sorrento, Italy, 23-26 August 1994, Eurotherm series 42 - EETI ed. Paris, 1995, pp. 260-226.

\section{Front face}



Figure 1 : Model used for the numerical computations: periodic cluster of slots. The front face is pulse heated (uniform energy density). Due to inherent symmetries, only half of a period is modelled.

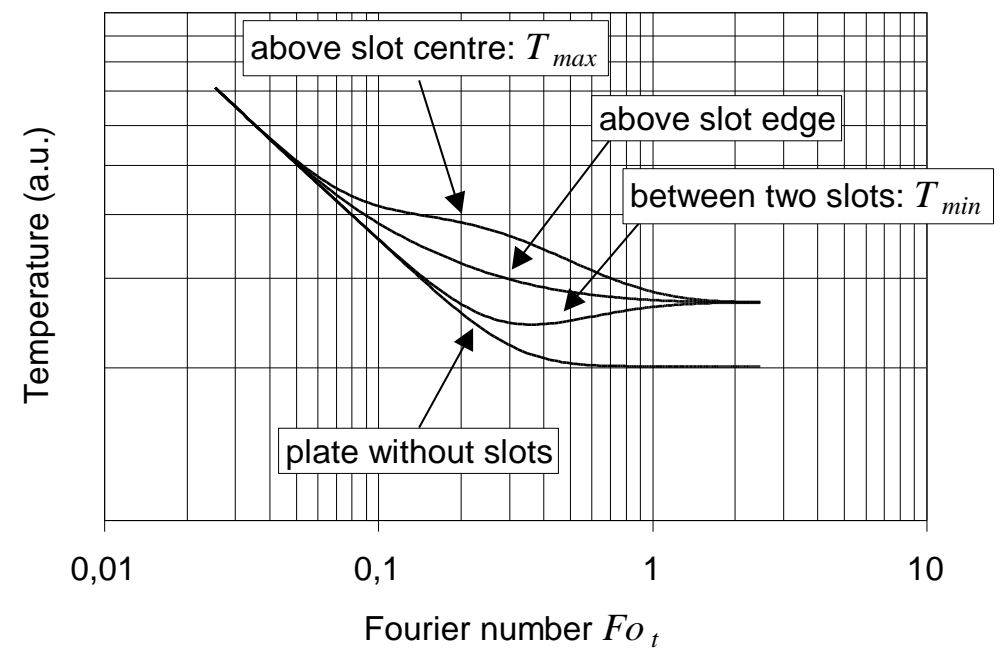

Figure 2 : Thermograms obtained on different parts of the target surface. Relative depth of the slots $l_{2} / l_{t}$ is 0.5 , spatial frequency $f_{t}$ is 0.3 . 

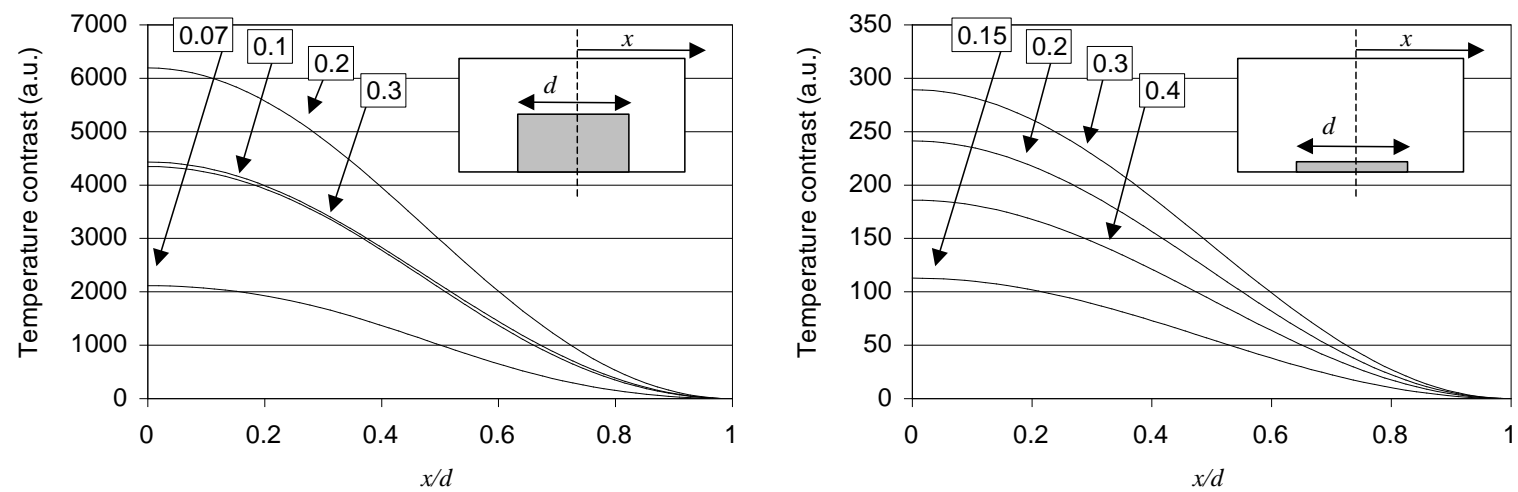

Figure 3 : Temperature contrast on the front surface at different times (the Fourier number is indicated on the curves). Spatial frequency of the target $f_{t}$ is 0.5 . Relative depth of the slots $l_{2} / l_{t}$ is 0.5 (left) and 0.1 (right).
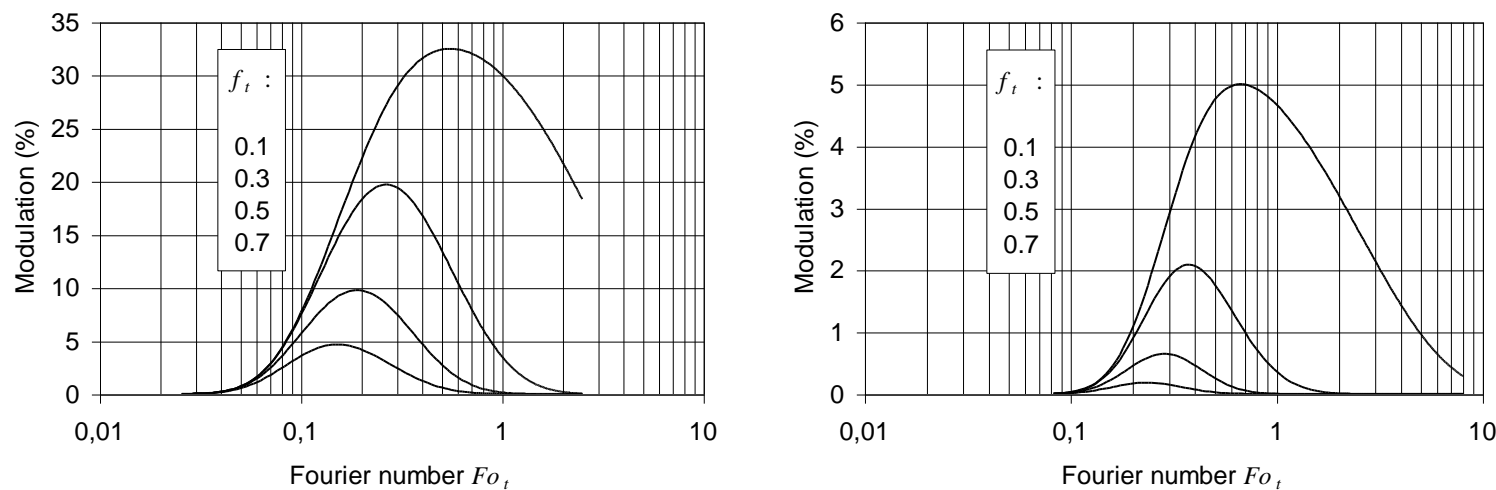

Figure 4 : Time evolution of the modulation for different spatial frequencies of the target. Relative depth of the slots $l_{2} / l_{t}$ is 0.5 (left) and 0.1 (right).


Figure 5 : Thermal modulation vs. spatial frequency at a time corresponding to a Fourier number $F_{o_{\mathrm{t}}}$ of 0.3 (left) and 0.5 (right). 


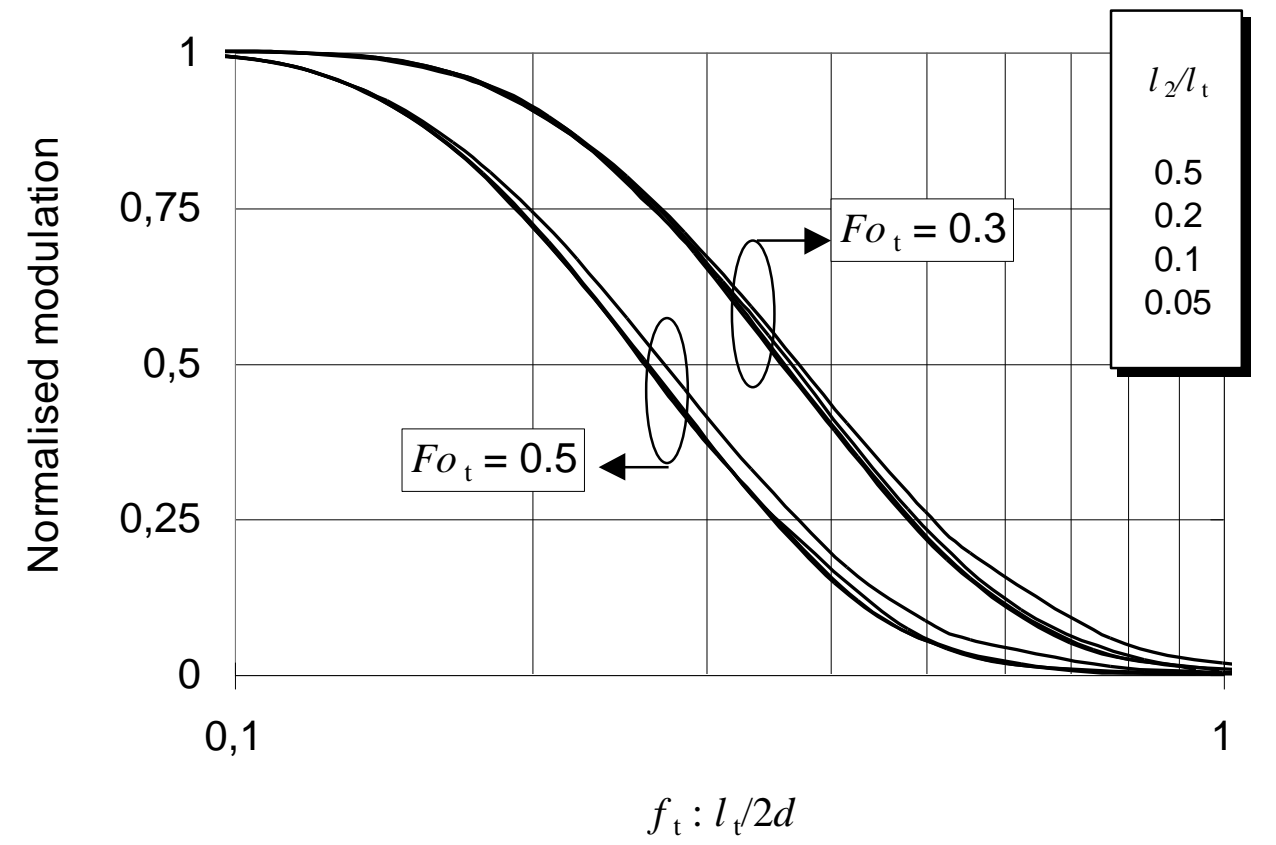

Figure 6 : Normalised thermal modulation vs. spatial frequency for two particular values of the Fourier number. For each curve bundle the relative depth of the slots $l_{2} / l_{t}$ is, from top to bottom: $0.5,0.2,0.1$ and 0.05 .

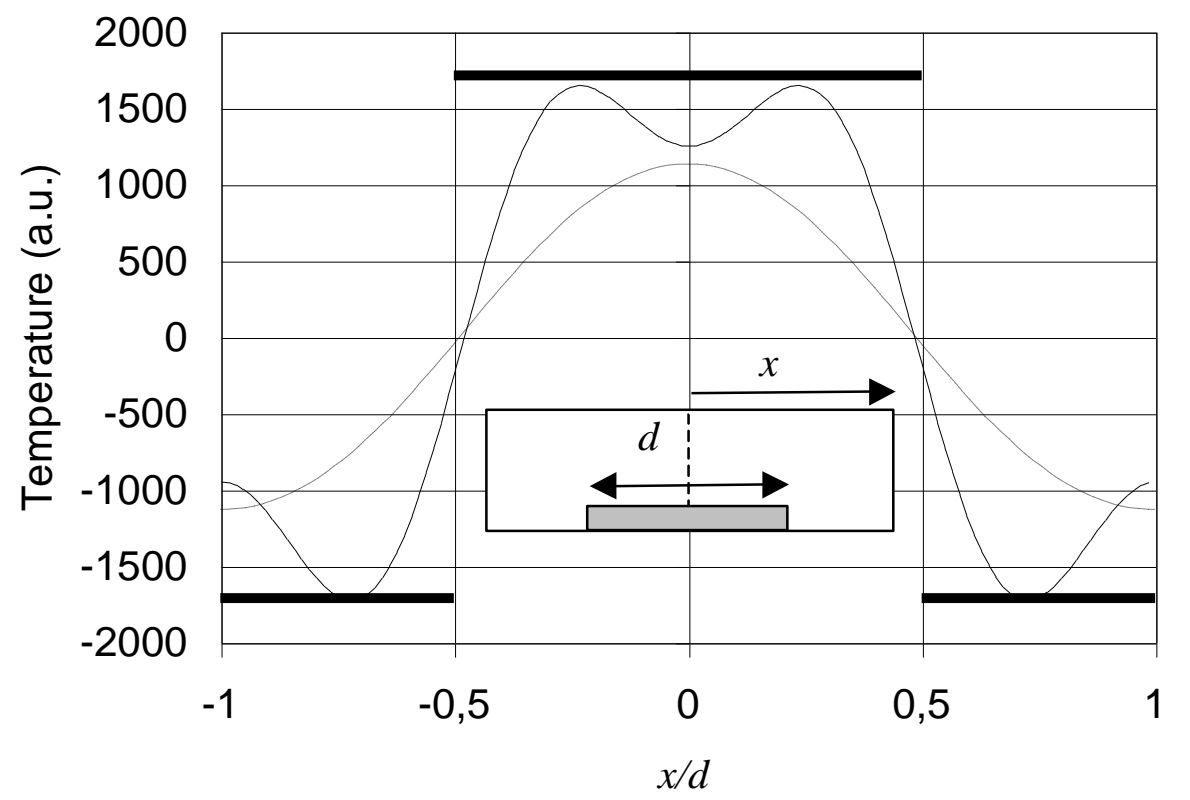

Figure 7 : Thermal profile (dot curve) at a time corresponding to $F o_{\mathrm{t}}=0.3$ in the case: $l_{2} / l_{t}=0.2$ and $f_{t}=0.3$. Inversion of the thermal profile using the TMTF (continuous line). The profile one would really obtain in the absence of lateral diffusion is indicated with thick horizontal lines. 